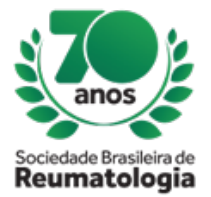

\title{
CHRONIC NONBACTERIAL OSTEOMYELITIS: REPORT OF SIX CASES
}

Gabriela Guimarães Moreira Balbi (UNIFESP, São Paulo, SP, Brasil), Daniela Petry Piotto (UNIFESP, São Paulo, SP, Brasil), Mateus Sabaini Venazzi (UNIFESP, São Paulo, SP, Brasil), Andre Aihara (UNIFESP, São Paulo, SP, Brasil), Artur Fernandes (UNIFESP, São Paulo, SP, Brasil), Claudio Arnaldo Len (UNIFESP, São Paulo, SP, Brasil), Maria Teresa Terrerri (UNIFESP, São Paulo, SP, Brasil)

\section{BACKGROUND}

Chronic nonbacterial osteomyelitis (CNO), alternatively known as chronic recurrent multifocal osteomyelitis (CRMO), is a rare autoinflammatory bone disorder that causes multifocal aseptic lytic lesions in bone biopsy. The course of the disease is caracterized by exacerbations and remissions, mostly affecting children and adolescents.

\section{MATERIALS AND METHODS}

Retrospective descriptive review of six cases of CNO in children and adolescents, treated at a specialized center, between 2010 and 2018.

\section{RESULTS}

Four (66\%) out of 6 patients were girls with mean age at evaluation of 15.5 years (range 11-20.4) and mean follow-up time of 5.1 years (range 1.5-10.2). Mean age of first symptoms was 10.3 years (range 0.715.3 ) and mean age at diagnosis was 12.8 years (range 9.5-16). The most affected sites were metaphysis and diaphysis of long bones. Median number of initial bony lesions was 2.8 (range 1-7) at onset and 3.5 (range 1-8) during the follow up. Two (33.3\%) patients had recurrence in clavicle, two in mandible and one (16.6\%) in temporomandibular joint. One (16.6\%) patient presented with fever and dyserythropoietic anemia, and was diagnosed as Majeed syndrome. Five (83.3\%) patients presented with arthralgia and bone pain, and two (33.3\%) had acute migratory polyarthritis. All patients had increased acute phase reactants and radiographic and magnetic resonance imaging alterations. All patients received NSAIDs therapy (indomethacin) with clinical remission in half of the cases. Three (50\%) patients received bisphosphonates (alendronate), and one took methotrexate associated with alendronate. All patients had good clinical response and two achieved clinical remission.

\section{CONCLUSION}

The awareness of characteristic features of CNO is important for an early diagnosis and can help avoiding unnecessary diagnostic procedures and prolonged antibiotic therapy. 